WSRC-TR $--90-275$

DE9 1006436

KEY WORDS: Embedded Atom Method

Interatomic Potentials

Computer Aided Materials

Analysis

Molecular Dynamics

RETENTION PERIOD: LIFETIME

\title{
Molecular Modeling of Metal Hydrides: II. Calculation of Lattice \\ Defect Structures and Energies Utilizing the Embedded Atom \\ $\operatorname{Method}(U)$
}

B y

R. J. Wolf and K. A. Mansour

ISSUED: December 1990

Westinghouse Savannah River Company

Savannah River Site

Aiken, South Carolina 29808

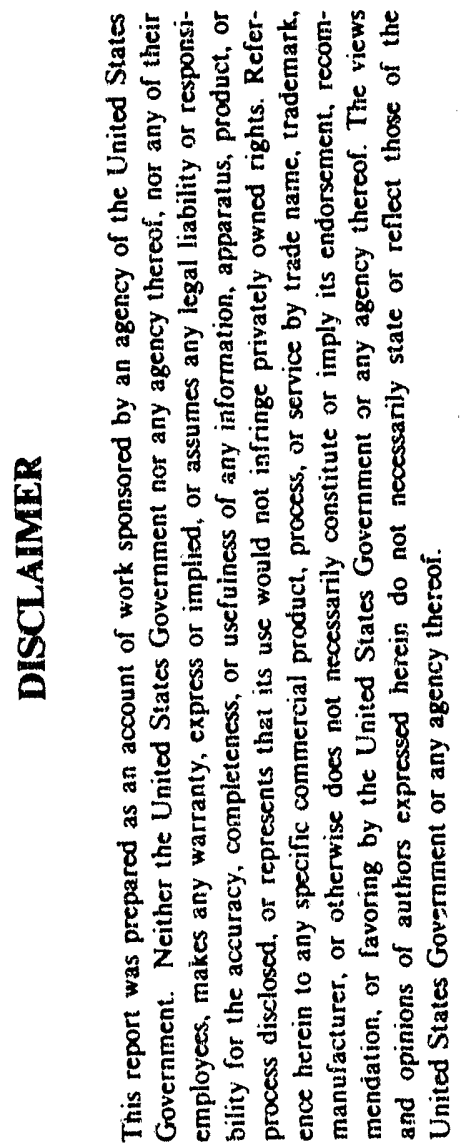

Prepared for the U. S. Department of Energy under Cuntract DE-AC09-89SR18035 


\section{DOCUMENT: WSRC-TR-90-275}

TITLE: Molecular Modeling of Metal Hydrides:

II. Calculation of Lattice Defect Structures and

Energies Utilizing the Embedded Atom Method(U)

TASK: QA TASK 89-053-0

QA TASK 89-053-A-0

\section{APPROVALS}

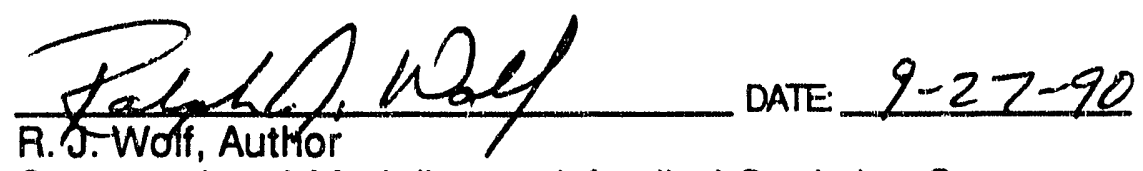

Computational Modeling and Applied Statistics Group

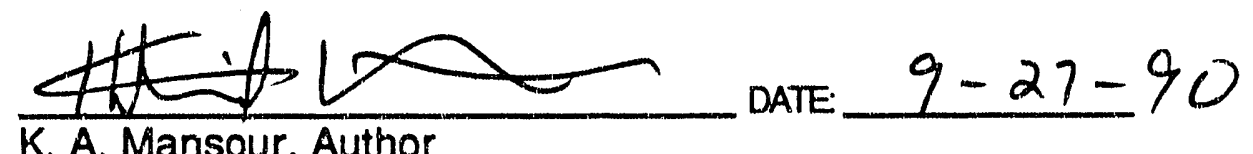

Computational Modeling and Applied Statistics Group

Meruef/ar fue

Hydrogen Technology Section

$\frac{\text { Ge Peyrey }}{\text { J. T. Pelfrey, Manager } 9-27-90}$

Computational Modeling and Applied Statistics Group

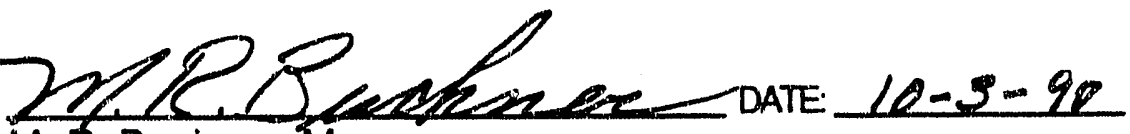

M. R. Buckner, Manager

Scientific Cornputations Section 
WSRC-TR-90-275

\title{
Molecular Modeling of Metal Hydrides: II. Calculation of Lattice Defect Structures and Energies Utilizing the Embedded Atom Method
}

\author{
By \\ R. J. Wolf and K. A. Mansour \\ Westinghouse Savannah River Company \\ Savannah River Laboratory \\ Nuclear Reactor Technology and Scientific Computations \\ Aiken, SC 29808
}

\begin{abstract}
Lattice defect structures and energies for palladium, nickel and aluminum computed include: single vacancy, self-interstitial, intrinsic stacking fault, coherent twin boundary and (100), (110), and (111) free surfaces. The importance of considering lattice defects in obtaining an accurate Embedded Atom Method (EAM) description of real materials, and the application of the EAM to the computation of lattice defect structures for paliadium, nickel and aluminum is discussed. The EAM functions developed in this study reproduce defect properties well and are suitable for future investigations of metal hydrides involving defect related structures.
\end{abstract}

\section{INTRODUCTION}

This report is the second in a series of documents describing our progress in modeling properties of metal hydrides. Reference to the initial report [WSRC-TR-90-156 or ref. 1] will be made, particularly to the methodology employed there. The present report focuses on two issues: The importance of considering lattice defects in obtaining an accurate Embedded Atom Method (EAM) description of real materials, and the application of such a description to the computation of lattice defect structures for palladium, nickel and aluminum.

Metal hydrides are vital SRS materials and will be used extensively for hydrogen/tritium storage and handling in the new Replacement Tritium Facility. Examples of such metals and metal alloys include palladium (Pd) and lanthanum-nickel-aluminum ( $\operatorname{LaNi}_{5-\mathrm{x}} \mathrm{Al}_{\mathrm{x}}, \mathrm{x}=0.15$ to 0.85 ). Metal hydrides absorb large quantities of hydrogen/tritium, and the hydrogen density in these materials can be much larger than that of liquid hydrogen. However, material degradation due to the ingrowth of helium, one of tritium's radioactive decay products, causes unrecoverable damage. Helium, although inert, tends to remain in the hydrides used at SRS once it is born. A clearer understanding of such a phenomenon is the goal of this work.

The main focus is to fundamentally understand hydrogen/tritium and helium behavior and their interactions in metal hydrides, with the intention to design better and more efficient tritium handling materials and facilities. As a by-product of this work, we will be able to apply our state-of-the-art techniques to study other problems involving hydrogen/tritium and helium in SRS materials. Examples include hydrogen and helium interactions in lithium-aluminum reactor targets, tritium reservoirs, and stainless steel reactor tanks. 


\section{DISCUSSION}

\section{Computational Methods}

In the section titled EAM Formalism, the EAM is reviewed as is the implementation at SRS of the method. Fitting EAM Functions presents details of the fitting procedure to obtain accurate EAM functions. Approximate Atomic Positions for Lattice Defects is concemed with defining unrelaxed defect structures. Unrelaxed structures are initial atomic positions for the energy minimization scheme employed to find a local minimum corresponding to the "predicted" defect structure. This is discussed further in the section titled Velocity Quench Energy Minimization Method..

\section{EAM Formalism}

The calculation of fundamental point and planar defects structures and energies presented here employ a form of potential closely related to the Embedded Atom Method work of Daw and Baskes [2]. Point defect structures investigated are single vacancy and self-interstitial. Planar defects studied include intrinsic stacking fault, coherent twin boundary, and the (100), (110), and (111) surfaces.

For a pure metal, the energy of an $\mathrm{N}$-atom system is

$$
E=\sum_{i=1}^{N} E_{i}
$$

where the energy of atom $i$ is given by

$$
E_{i}=G\left(\rho_{i}\right)+U\left(r_{i j}\right) .
$$

Here $r_{i j}$ is the distance between the atoms $\mathrm{i}$ and $\mathrm{j}, \mathrm{U}$ is a pair interaction potential, $r_{\mathrm{i}}$ is the electron density at atomic site i due to remaining $(\mathbb{N}-1)$ atoms,

$$
\rho_{i}=\sum_{j \neq i}^{N} \rho\left(r_{i j}\right) .
$$

The embedding energy, $G\left(r_{\mathfrak{i}}\right)$, is interrireted as the energy resulting from embedding atom $i$ in an electron gas of density $r_{\mathrm{i}}$. As describel in Ref. 1 , atomic electron densities, $r\left(\mathrm{rij}_{\mathrm{ij}}\right)$, are taken from the Hartree-Fock wave functions of Clementi and Roetti [3] and $U\left(r_{i j}\right)$ is defined by the Morse potential,

$$
U\left(r_{i j}\right)=D\left\{\exp \left[-\beta\left(r_{i j}-r_{o}\right)\right]^{2}-2 \exp \left[-\beta\left(r_{i j}-r_{o}\right)\right]\right\}
$$

where $\mathrm{D}, b$ and $\mathrm{r}_{\mathrm{O}}$ are adjustable pararneters.

In this report, the pair potential and the density functions were modified by forcing their values and derivatives to zero at a specified cut-off distance $r_{c}$. As in past EAM studies [4] the cut-off distance was chosen between the third and fourth neighbor distances in the face-centered cubic 
(FCC) structure. This is accomplished by multipling the pair and density functions, $F(r)$, by a switching function, $S(r)$, to obtain a new function, $f(r)$ which smoothly goes to zero at $r_{c}[5]$. That is;

$$
f(r)=S(r) F(r)
$$

where $S(r)$ is defined by

$$
S(r)=\left\{\begin{array}{cc}
\exp \left(\frac{\eta}{r-r_{c}}\right) & r<r_{c} \\
0 & r \geq r_{c}
\end{array}\right.
$$

and $\eta$ is a scaling factor which controls the rate of the cut-off. An $\eta$ of 0.10 was used in our calculations while $r_{c}$ was set to $1.31 a_{0}$, where $a_{0}$ is the lattice constant for the material of interest. The value $1.31 a_{0}$ restricts the interactions to third neighbors in the FCC structures described here, and would restrict interaction to second neighbors in the body-centered cubic $(\mathrm{BCC})$ structures and fourth neighbors in the hexagonal close packed (HCP) structures.

\section{Fitting EAM Functions}

The EAM functions were obtained as described in our initial report [1], but with two differences. First, the number of neighbor shells considered in the initial report was four, and we consider only three as detailed above. This was done because it is difficult to reproduce stacking fault energies with the EAM and the functions chosen when the interactions extend beyond third neighbor interactions [6]. The problem appears to be associated with a cut-off value of $1.354 \mathrm{a}_{0}$ or the fifth nearest neighbor distance in the HCP structure. In the region of the stacking fault four planes have the neighbor shells with distances of $0.7071 \mathrm{a}_{0}, 1.000 \mathrm{a}_{0}, 1.1547 \mathrm{a}_{0}, 1.2247 \mathrm{a}_{0}, 1.354 \mathrm{a}_{0}$, and $1.4142 \mathrm{a}_{0}$ and have $12,6,1,21,6$, and 9 atoms per shell, respectively. The values of $1.1547 \mathrm{a}_{0}$ and $1.354 \mathrm{a}_{0}$, are unique to the HCP structure while the others are members of both HCP and FCC structures.

The second difference is that the EAM functions were fit to the elastic constants $\left(C_{11}, C_{12}\right.$, and $\mathrm{C}_{44}$ ), unrelaxed single vacancy formation energy, $\mathrm{E}_{u v f}$, and the unrelaxed intrinsic stacking fault energy, $E_{u s f}$. With the exception of $E_{u s f}$, the numerical evaluation of these were discussed 
previously [1]. In the unrelaxed intrinsic stacking fault there are four equivalent planes of atoms which do not have an FCC nearest neighbor arrangement and the energy per unit area in the plane is:

$$
E_{u s f}=4\left\{\sum_{n} w_{n}^{s f} E_{n}^{s f}-\sum_{n} w_{n}^{f c c} E_{n}^{f c c}\right\} / \frac{\sqrt{3}}{4} a_{o}^{2}
$$

where the sums are over nearest neighbor shells, $W_{n}$ is the number of atoms in the nth shell and $E_{n}$ is the energy contributed by an atom in the nth shell. The shells are for the FCC neighbor list (fce) or the stacking fault neighbor list (sf). The equation above provides a computationally efficient means of calculating the unrelaxed stacking fault energy (with no relaxation of the atomic positions). A derivation of this equation is presented in Appendix A.

Numerical fitting of the Morse parameters, $\mathrm{D}, b$ and $\mathrm{r}_{\mathrm{O}}$, was achieved utilizing a simplex search procedure [7]. The function minimized was the sum of the deviations squared multiplied by a weight squared where the deviation is the difference between the experimental data and the calculated property. A variety of "reasonable" fits can be obtained depending on the initial values of the parameters and the set of weights used. In addition, such a fit may not yield an acceptable $G(r)$ whose desirable properties include a minimum and upward curvature at higher $r$ values. An acceptable $\mathrm{G}(r)$ results from parameters within the approximate ranges of $\mathrm{D}=0.1$ to $2.0 \mathrm{eV}, b=$ 0.9 to $2.0 \AA^{-1}$ and $r_{0}=1.7$ to $3.0 \AA$ for the metals studied.

The embedding energy, $G(r)$, is determined via an inversion procedure [4] utilizing an equation of state (EOS) due to Rose, et. al. [8], which insures that we reproduce the experimentally deterrnined values of the cohesion energy, $\mathrm{E}_{\mathrm{c}}$, the bulk modulus, $\mathrm{B}$, and the lattice constant, $a_{0}$. $\mathrm{G}(r)$ defined in this manner is numerical in nature. An analytic form is desirable for rapid force evaluation. Therefore, the inversion method was used to obtain $G(r)$ data for densities associated with a lattice expansion of $2-5 \%$ and compression of $10-20 \%$. The data was numerically fit to an analytical form using a non-linear least squares method. The EOS equations by Rose, et. al. [8] were fit (and therefore only accurate) over this range. The analytical form chosen was

$$
G_{a}(\rho)=A \rho \exp (-a \rho)+B \rho^{3} \exp (-b \rho)+C \rho \exp (-c \rho) \text {. }
$$

A variation of this form has been used by Truong, et. al. [5]. The analytical form, $G_{a}(r)$, was compared to the $\mathrm{G}(r)$ obtained by inversion of the EOS, $\mathrm{G}_{\mathrm{ens}}(r)$, by recomputing the material properties used to obtain the fit.

\section{Approximate Atomic Positions for Lattice Defects.}

Initial positions for atoms in a rectangular box with periodic lattice conditions can be generated by replicating a unit box consisting of a minimal number of atoms. For an FCC arrangement this involves translational replication along cartesian axis of a cube containing four atoms where the repeat distance in all three directions $(x, y, z)$ is given by the lattice parameter, $a_{0}$. Slicing this lattice perpendicular to the z-axis exposes a (100) face of the FCC crystal lattice. The (110) and (111) FCC faces can be exposed by the same slicing if the lattice is replicated using different unit 
cells which are no longer cubic but whose axes are still mutually orthogonal. The cell dimensions or replication lengths of $\mathrm{a}, \mathrm{b}$, and $\mathrm{c}$ lie along the $\mathrm{x}, \mathrm{y}$, and $\mathrm{z}$ axes, respectively, and the atomic positions in the unit cell are given in Table I. The procedure to generate the atomic cartesian positions for an array of atoms is expressed as

$$
\begin{aligned}
& X_{m}=X_{i}^{O}+(j-1) a ; \quad 0 \leq j \leq J \\
& Y_{m}=Y_{i}^{O}+(k-1) b ; \quad 0 \leq k \leq K \\
& Z_{m}=Z_{i}^{o}+(l-1) c ; \quad 0 \leq l \leq L
\end{aligned}
$$

where a new set of $\left(X_{m}, Y_{m}, Z_{m}\right)$ is produced for every combination of $j, k$, and $l$ within the limits and is applied to every atom (index by $\mathrm{i}$ ) in the unit cell whose positions are denoted $\left(\mathrm{X}_{\mathrm{i}}{ }^{0}, \mathrm{Y}_{\mathrm{i}}{ }^{\mathrm{O}}\right.$, $\mathrm{Z}_{\mathrm{i}}{ }^{\mathrm{O}}$ ). The box containing the atoms has $(\mathrm{x}, \mathrm{y}, \mathrm{z})$ dimensions of $\mathrm{Ja} \times \mathrm{Kb} \times \mathrm{Lc}$ with atomic coordinates suitable for periodic conditions. If periodic conditions are removed for the $x y$ plane (i.e.; the z-coordinate) then two free surfaces are exposed each with a surface area of Ja $\mathbf{x ~ K b}$.

Point defects can be easily created by generating an FCC lattice of atoms (as in the (100) case above) and adding an atom to an octahedral interstitial site to create a self interstitial or deleting an atom to create a single vacancy. Since all boundaries should be periodic it should be of no consequence which octahedral hole is filled or which atom is removed.

Creation of a planar defect is more complicated [9]. An FCC lattice consists of close-packed planes (i.e.; (111) planes or octahedral planes) stacked and labeled $A B C A B C A B C A B C . .$. where the $A, B$, or $C$ designation refers to the plane's displacement in the $x y$ plane. That is, all $A$ planes have the same xy coordinates and similarly for $B$ and $C$ planes. An intrinsic stacking fault is created when the stacking pattern gets out of sequence and a plane is skipped (or removed); for example, $A B C A B C \mid B C A B C .$. where the vertical bar marks the skipped plane. To obtain atomic positions and preserve periodic lattice conditions, a FCC lattice can be generated with the (111) prescription given above and an A plane can be removed from the center (an xy plane) of the array of atoms and placed on top of the C plane exposed on the top,

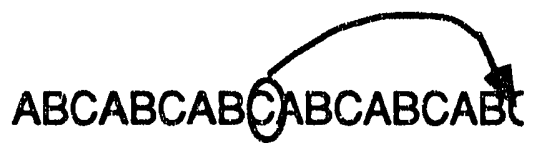

where a gap equal to twice the distance between planes will remain. The final step is to shift all the planes above the gap down a distance to obtain the normal spacing. Periodic boundary conditions in the z-coordinate would produce a periodic infinite array of stacking faults, therefore the condition of periodic boundaries is removed for the z-coordinate leaving a stacking fault and two (111) surfaces exposed. Thus, calculation of the stacking fault energy from the array of atoms generated requires the computation of the (111) surface energy.

A coherent twin boundary can be obtained in a similar fashion to the stacking fault. The stacking in a coherent twin boundary is 
where the stacking follows the sequence $\mathrm{ABC}$... from either top or bottom and finally meet, only having to share a plane (in this case $C$ ). The generation of the array of atoms is done by filling an array of one half the desired length (where $L$ must be even) in the z-direction and then reflecting all the atoms about the top plane of the generated cell (leaving a gap again in the center) and moving the top plane to fill the gap in the center. In this case, the periodic boundary condition could apply as in the stacking fault case, but as with the stacking fault the $z$-coordinate is made free of periodicity by creating two surfaces. The energy resulting from this array of atoms has contributions of a twin boundary and two (111) surfaces.

In all cases, the size of the array of atoms ( $\mathrm{J}$ by $\mathrm{K}$ by $\mathrm{L}$ unit cells) is determined by convergence. That is, the defect energy is taken in the limit of large $J, K$, and $L$ where energies and relative positions of the atoms farthest from the defect should reflect that of a bulk phase atom.

\section{Velocity Quench Energy Minimization Method.}

The atomic structures created in the previous section are labeled approximate because atoms in the region of the defects will move, or relax, in order to decrease the total energy of the system. This motion is governed by the forces exerted on the atoms and Newton's law of motion $\vec{F}=m \vec{a}$, where $\vec{F}$ is the force, $\mathrm{m}$ the mass of the atom and $\vec{a}$ is the acceleration. The force on an atom can be calculated by

$$
\vec{F}=-\frac{d E}{d r} \cdot \hat{r}
$$

where $\hat{r}$ is the unit vector in the direction of the atom position vector, $\vec{r}$. Newton's law of motion for atom $i$ can be written in the form

$$
\ddot{\vec{r}}=-\frac{1}{m_{i}} \vec{F}_{i}
$$

where the acceleration has been replaced by

$$
\ddot{a}=\frac{d^{2} \vec{r}}{d t^{2}}=\ddot{\vec{r}}
$$


For the EAM the force on an atom is given by

$$
\vec{F}_{i}=-\sum_{j \neq i}^{N}\left\{G_{i}^{\prime} \rho_{j}^{\prime}+G_{j}^{\prime} \rho_{i}^{\prime}+U^{\prime}\right\} \cdot \hat{r}_{i j}
$$

where the index $i$ (or $j$ ) implies evaluation at atom $i$ (or $j$ ) and the primes indicate derivatives with respect to $r$ (or $r$ in the case of $\mathrm{G}$ ) [10]. 'There are $3 \mathrm{~N}$ equations of motions for an $\mathrm{N}$ atom system.

The velocity quenched energy minimization method (VQEMM) [11] consists of the integration of the $3 \mathrm{~N}$ equations of motion and monitoring each of the $3 \mathrm{~N}$ cartesian velocities at each step of the numerical integration. Whenever the force in a given direction has the opposite sign of the velocity, that component of the velocity is set to zero and integration continues until atomic motion ceases or the total energy of the system converges to a fixed value. At this point, an equilibrium structure of the atoms has been located in the $3 \mathrm{~N}$-dimensional energy function.

By design, the local minimum determined by this method is the nearest minimum to the initial atomic positions as directed by their energy gradient. To insure reduction of local symmetry in the forces and possibly a saddle point (an unstable equilibrium point) in the hyperspace the initial atomic cartesian coordinates were randomized by addition of a uniform randorn displacement between the bounds of +0.05 and $-0.05 \AA$.

\section{Results and Discussion}

EAM functions have been developed for palladium ( $\mathrm{Pd}$ ), aluminum ( $\mathrm{Al}$ ) and nickel (Ni), and comparisons are presented here utilizing these functions with experimentally determined or derived data. The Morse function parameters, $\mathrm{D}, \mathrm{r}_{0}$, and $b$, obtained in the fit are listed in Table II. In addition, the parameters describing the analytic form of the embedding function, $\mathrm{G}_{\mathrm{a}}(r)$, are listed. In Table III, we present a comparison of experimental values used in the fit to those computed using both $\mathrm{G}_{\text {eos }}(r)$ and $\mathrm{G}_{\mathrm{a}}(r)$. Recall that $\mathrm{G}_{\mathrm{a}}(r)$ is the desired form of the embedding function to be utilized in the VQEMM and $G_{\text {eos }}(r)$ is the direct result of the fit utilizing the inversion of the equation of state.

In Table III, a comparison is made with experimental values [12] and computational [4,13-15] values where possible. The computational studies do not attempt to fit the intrinsic stacking fault which proved to be a significant restriction. EAM functions developed previously for palladium, which fit eiastic constants extremely well, gave unphysical (negative) stacking fault energies when tested. To achieve reasonable stacking fault energies a small trade-off in the accuracy of elastic constants must be made (a similar example of this exists in the literature). In an attempt [16] to fit EAM functions to the stacking fault energy in nickel, the fit to elastic constants was constrained by fitting $\mathrm{C}_{44}$ and $\left(\mathrm{C}_{11}-\mathrm{C}_{12}\right) / 2$. This procedure concentrates on obtaining only the difference between $C_{11}$ and $C_{12}$, not their absolute magnitudes. For our application, argument wou d support obtaining reasonable defect structure and energies at the expense of highly accurate pure crystalline elastic properties.

As a test of the EAM functions developed we have computed the relaxed vacancy formation, selfinterstitial formation, (100), (110) and (111) surfuce, intrinsic stacking fault, and coherent twin boundary energies. The energy minimization procedure consisted of an initial configuration of atoms with a structure close to the expected defect structure which follows a modified classical trajectory path, see Section IID. 
Point defect energies are calculated by taking the difference between final relaxed energy of the system containing the defect and the energy of a reference state with no defect, but an equal number of atoms [17-18]. All calculations are performed at constant volume. Surface energies can be calculated by taking the difference as done in the point defects and dividing by the total surface area. Remember that the surfaces are created by removing the z-component periodic boundary and there are two free surfaces of the same area. Stacking fault and twin boundaries cases are similar to the free surfaces except their total energies are due to two free (111) surfaces and the planar defect. To calculate their energies one can simply use a (111) surface calculation with the same size of computational lattice as a reference state. The defect. energy is then the difference between the energy of the system with the defect (and two (111) surfaces) and the reference state, all divided by the surface area of the defect (same as one (111) surface). All calculations involving surfaces or boundaries have $x$ and $y$ periodic boundaries fixed [18].

Convergence tests were performed, using the palladium functions, to determine the appropriate cell size or number of atoms needed to remove boundary effects. Table IV shows the results of varying the size of the computational cells. The results iridicate that a cell containing 500 atoms is sufficient for computing defert energies (to three significant figures). In the case of (110) surfaces, energy convergence is good even in the case of $L=4$ or eight planes of atoms and in the (111) case of $L=4$ or 12 planes of atoms. For stacking faults and twin boundaries the cell is expanded to twice the thickness (in the z-direction) in order to isolate the planar defect from the two free (111) surfaces exposed.

Unrelaxed vacancy formation energies, $E_{u v f}$, were optimized in the fitting process with the assumption that relaxation was small and using an approximate formula [1]. This is seen numerically in Table $\mathrm{V}$. Relaxed vacancy formation energies, $\mathrm{E}_{\mathrm{vf}}$, are in good agreement with the experimental values. Self interstitial formation energies are in good agreement with past EAM calculations[4b]. Experimental values are not available. Surface energies for Ni are in excellent agreement with experiment [19]. Pd and Al surface energies follow the trend of the surface tension meisurements [19].

Intrinsitic stacking fault energies, $\mathrm{E}_{\mathrm{sf}}$, arc in the range of the experimental values, see Table $\mathrm{V}$. This assessment is made with caution since experimental values vary wildly. For example, in nickel [20] values range from $120-150 \mathrm{ergs} / \mathrm{cm}^{2}$ with no apparent trend with time, although the value used of $125 \mathrm{ergs} / \mathrm{cm}^{2}$ appears to be the most accepted. The value of $108 \mathrm{ergs} / \mathrm{cm}^{2}$ is acceptable.

An intrinstic stacking fault can be viewed as two coherent twin boundaries separated by a close packed plane of atoms. Therefore, from a hard sphere interaction model one expects that the energy of a coherent twin boundary, $E_{t b}$, is approximately one-half of the intrinstic stacking fault energy [21]. This fact is borne out in our calculations, see Table V.

Structural relaxation is small in most the defects studied. The self-interstitial suructure agrees with the accepted dumbbell like arrangement (along the $\langle 100\rangle$ direction) observed previously [24] to be the most stable orientation. The extent of surface (planar) relaxation is given in Table VI for both 
the free surfaces and planar defects. The extent of relaxation is measured as the change in the interplanar stacking distance from the bulk. In the case of planar defects, this is the extent of the shift for planes near the defect and continuing away from the defect. In the case of the intrinsic stacking fault, the planar spacings $z_{12}$ and $z_{23}$ are defined as

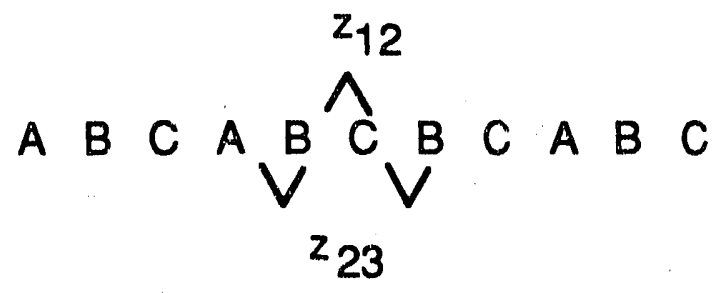

and for the coherent twin boundary as

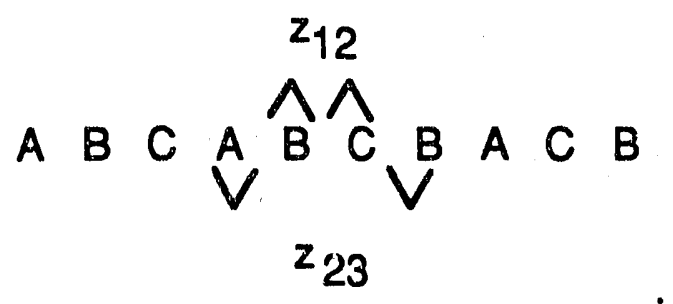

In the stacking fault the symmetry plane lies between two atomic planes, whereas, in the twin boundary it is coincident with an atomic plane. The surface structures determined here account for relaxation but not surface reconstruction, which is reserved for cases where a surface is annealed and then quenched. Inter-planar relaxations are in agreement but characteristically smaller than the range observed experimentally [22] for these materials.

\section{Conclusions and Summary}

The EAM functions developed in this study reproduce defect properties well and are suitable for future investigations of metal hydrides involving defect related structures. Molecular modeling efforts involving palladium as a metal hydride appear very promising. The functions for nickel and aluminum (both FCC) metals, represent a start at describing the lanthanum-nickel-aluminum ( $\mathrm{LaNi}_{5-\mathrm{x}} \mathrm{Al}_{\mathrm{x}}, \mathrm{x}=0.15$ to 0.85 ) system. Lanthanum, an HCP metal, may not fit well within the EAM approach as a pure metal but may alloy in a sufficient manner. This will require further EAM development.

Helium-metal interactions are currently being developed to investigate helium bubble formation, which are thought to nucleate at most defects (point defects, twins, stacking faults and grain boundaries). Hydrogen-metal and hydrogen-helium interactions, also being developed, ave necessary to understand the interaction of hydrogen present in metal hydrides. These new functions enable simulation of the behavior and properties of palladium containing hydrogen and helium. 


\section{ACKNOWLEDGEMENTS}

We would like to express our gratitude to Drs. M. W. Lee, E. A. Clark, M. R. Louthan of the Savannah River Laboratory, and Dr. S. M. Folies of the Sandia National Laboratory, Livermore for many useful discussions. 


\section{Appendix A}

The urrelaxed stacking fault energy can be calculated by using a stummation over the neighbor list of the atoms in the region of the fault. A stacking fault exists when a plane is skipped in the normal FCC stacking sequence of (111) planes. The stacking in the immediate vicinity of the fault appears to be that of an HCP structure. In the region of the stacking fault four planes have the neighbor shells with distances similar to tie FCC and HCP structures. All other atoms have FCC neighbor lists. The neighbor lists for these cases are

\begin{tabular}{clrrr}
\hline & & \multicolumn{3}{c}{ Number of Atoms in Shell } \\
Shell & Distance & FCC & HCP & Stacking Fault \\
\hline 1 & $0.7071 \mathrm{a}_{\mathrm{O}}$ & 12 & 12 & 12 \\
2 & $1.000 \mathrm{a}_{0}$ & 6 & 6 & 6 \\
3 & $1.1547 \mathrm{a}_{0}$ & 0 & 2 & 1 \\
4 & $1.2247 \mathrm{a}_{0}$ & 24 & 18 & 21 \\
5 & $1.354 \mathrm{a}_{0}$ & 0 & 12 & 6 \\
6 & $1.4142 \mathrm{a}_{0}$ & 12 & 6 & 9 \\
\hline
\end{tabular}

The neighbor lists begin to differ beyond the distance of $1.1547 \mathrm{a}_{0}$. Neighbor lists can be generated by constructing the desired lattice, as described in section IIC, and computing interatomic distances.

The energy of an atom with an FCC neighbor list is given by

$$
\sum_{n} W_{n}^{f c c} E_{n}^{f c c}
$$

while an atom in one of the four planes with the stacking fault neighbor list is

$$
\sum_{n}^{s f} E_{n}^{s f}
$$

where the sums are $\sigma$, nearest neighbor shells, $W_{n}$ is the number of atoms in the nth shell and $E_{n}$ is the energy contributed by an atom in the nth shell. The shells are for the FCC neighbor list (fcc) or the stacking fault neighbor list (sf).

The unrelaxed stacking fault energy is four times (one for each plane) the energy required to convert a normal FCC lan'ice arom into an atom in a stacking fault plane divided by the area of an atom in the (111) plane:

$$
E_{u s f}=4\left\{\sum_{n} w_{n} s E_{n}^{s f}-\sum_{n} w_{n}^{f c c} E_{n}^{f c c}\right\} / \frac{\sqrt{3}}{4} a_{o}^{2} .
$$




\section{REFERENCES}

1 K. A. Mansour and R. J. Wolf, WSRC-TR-90-156.

2 M. S. Daw and M. I. Baskes, Phys. Rev. Lett. 50, 1285 (1983).

3 E. Clementi and C. Roetti, Atomic and Nuclear Data Tables 1S, 177 (1974).

4 a) S. M. Folies, Phys. Rev. B 32, 7685 (1985); and b) S. M. Folies, M. I. Baskes, and M. S. Daw, Phys. Rev. B 33, 7983 (1986).

5 T. N. Truong, D. G. Truilur and B. C. Garrett, J. Phys. Chem. 93, 8227 (1989).

6 S. M. Folies, private communication.

7 numerical method implemented utilizing UMPOL from IMSL User's Manual Math/Library version 1.0 (IMSL, Houston, 1987) p. 831-834.

8 J.H. Rose, J.R. Smith, F. Guinea, and J. Ferrante, Phys. Rev. B 29, 2963 (1984).

9 J.P. Hirth and J. Lothe, Theory of Disslocations, (McGraw-Hill Book Co.,New York, 1968), pp. 289.

10 M.S. Daw and M.I. Baiskes, Phys. Rev. B 29, 6443 (1984).

11 also referred to as localized damping method, see J. R. Beeler, Jr., Radiation Effects Computer Experiments, (North-Holland Publishing Co., New York, 1983), pp. 237.

12 a) G. Sirmmons and H. Wang, Single Cryta' Elastic Constants and Calculated Aggregate Properties: A Handbook (MIT Press, Cambriage, 1971); b) Y. A. Kraftmaker and G. P. Strelkov, in Vacancies and Interstitials in Metals, edited by A. Seeger, D. Schumacher, W. Schilling, and J. Diehl (North Holland, Amsterdam, 1970), p.59; c) W. Wycisk and M. Feller-Kniepmeier, J. Nucl. Mater. 69\&70, 616 (1978); d) S. Koehler, in Vacancies and Interstitials in Metals, edited by A. Seeger, D. Schumacher, W. Schilling, and J. Diehl (North Holland, Amsterdani, 1970), p. 175.

13 S. M. Foiles and M. S. Daw, J. Mater. Res. 2, 5 (1987).

14 S. P. Chen, A. F. Voter, R. C. Albers, A. M. Boring, and P. J. Hay, J. Mater. Res. 5, 955 (1990); and S. P. Chen, D. J. Srolovitz, and A. F. Voter, J. Mater. Res. 4, 62 (1989).

15 D. J. Oh and R. A. Johnson, J. Mater. Res, 3, 471 (1988).

16 M. I. Baskes and M. S. Daw, to be published in Procedings of the 4th International Conference on Hydrogen Effects on Material Behavior, Jackson Lake Lodge, WY, September 1990).

17 J. M. Harder and D. J. Bacon, Phil. Mag. A 54, 651 (1986); and C. C. Matthai and D. J. Bacon, J. Nucl. Mater, 114, 22 (1983).

18 Embedded Atom Workshop Notes, August 1989, Sandia National Laboratories, Livermore, $C$. 
19 surface energies for Ni see: a) P. S. Maiya and J. M. Blakely, J. Appl. Phys. 38, 698 (1967); b) E. R. Hayward and A. P. Greenough, J. Inst. Metals 88, 217 (1960); c) F. A. Clark, R. Yeske and H. K. Birnbaum, Metall. Trans. A 11A, 1903 (2980); and d) R. M. Digilov, S. N. Zadumkin, V. K. Kumykov, and Kh. B. Khokonov Phys. Met. Mettall. 41, 68 (1976); for Pd see e) W. R. Tyson and W. A Miller, Surf. Sci. 62, 267 (1977); for Al see e) H. Jnnes, Metal Sci. J. 5, 15 (1971).

20 stacking faults for Pd see: a) I. L. Dillamore and R. E. Smallman, Phil. Mag., 12, 191 (1965); for Ni see: b) C. B. Carter and S. M. Holmes, Phil. Mag. 35, 1161 (1977); for Al see c) P. S. Dobson, P. J. Goodhew, and R. E. Smallman, Phil. Mag. 16, 9 (1967).

21 see pg.294 of ref. 9.

22 A. Seeger, E. Mann, R. v. Van, J. Phys. Solids 23, 639 (1962).

23 see pp. 237 of ref. 11.

24 H. B. Nielsen, J. N. Anderson, L. Petersen and D. L. Adams, J. Phys. C 14, L1113 (1982) and J. Phys. C 17, 173 (1985); J. R. Noonan and H. L. Davis, Phys. Rev. B 29, 4349 (1984); D. L. Adams, L. E. Petersen and C. S. Sorensen, J. Phys. C 18, 1753 (1985); and S. M. Yalisove, W. R. Graham, E. D. Adams, and T. Gustafsson, Surf. Sci. 171, 400 (1986). 


\section{Table I}

Definition of unit celis used to generate surfaces.

(100) faces

$a=b=c=a_{0}$

$\begin{array}{cccc}\text { atom } & \mathrm{X}^{\mathrm{O}} & \mathrm{Y}^{\mathrm{0}} & \mathrm{Z}^{\mathrm{0}} \\ 1 & 0.0000000 & 0.0000000 & 0.0000000 \\ 2 & 0.5000000 & 0.5000000 & 0.0000000 \\ 3 & 0.5000000 & 0.0000000 & 0.5000000 \\ 4 & 0.0000000 & 0.5000000 & 0.5000000\end{array}$

(110) faces $a=\frac{1}{\sqrt{2}} a_{0} \quad b=\frac{1}{2} a_{0} \quad c=\frac{1}{\sqrt{2}} a_{0}$

$\begin{array}{cccc}\text { atom } & \mathrm{X}^{0} & \mathrm{Y}^{0} & \mathrm{Z}^{0} \\ 1 & 0.0000000 & 0.0000000 & 0.0000000 \\ 2 & 0.5000000 & 0.5000000 & 0.5000000\end{array}$

111) faces $a=\frac{1}{\sqrt{2}} a_{0} \quad b=\sqrt{\frac{3}{2}} a_{0} \quad c=\sqrt{3} a_{0}$

$\begin{array}{cccc}\text { atcm } & X^{0} & Y^{0} & Z^{0} \\ 1 & 0.0000000 & 0.0000000 & 0.0000000 \\ 2 & 0.5000000 & 0.5000000 & 0.0000000 \\ 3 & 0.5000000 & 0.1666667 & 0.3333333 \\ 4 & 0.0000000 & 0.6666667 & 0.3333333 \\ 5 & 0.0000000 & 0.3333333 & 0.6666667 \\ 6 & 0.5000000 & 0.8333333 & 0.6666667\end{array}$


Table II.

EAM Function Parameters.

EOS data

$\mathrm{Pd}$

$\mathrm{Ni}$

$\mathrm{Al}$

$\mathrm{E}_{\mathrm{c}}(\mathrm{eV})$
$\mathrm{B}\left(\mathrm{eV} \AA^{-3}\right)$
$\mathrm{a}_{0}(\AA)$

3.91

4.45

3.36

1.127

3.52

0.493

3.89

4.05

Morse function parameters

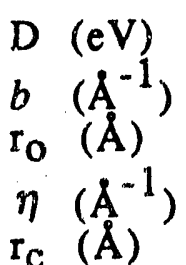

0.36937

0.6645

0.13113

1.3485

1.9372

0.99808

2.2649

2.75570

$\eta\left(\AA^{-1}\right)$

0.10

5.1

0.10

0.10

4.62

5.3

$\mathrm{G}_{\mathrm{a}}(r)$ parameters
A
$-122.006$
$-76.2136$
$-181.685$
74.0547
34.8861
44.0935
B
$9.95144 \times 10^{3}$
$2.04296 \times 10^{3}$
6.15084
$-91.2633$
$-76.2556$
38.2764
$9.67593 \times 10^{5}$
118.811
$-181.685$
31.9016
44.0935 
Table III.

Pure metal properties used to determine EAM functions.

The first two numbers given are from this work, the top number is the calcuated using $G_{a}(r)$, the second using $G_{\text {eos }}(r)$.

\begin{tabular}{|c|c|c|c|c|}
\hline & $\mathbf{P d}$ & $\mathrm{Ni}$ & Al & reference \\
\hline $\mathrm{C}_{11}$ & $\begin{array}{l}2.37 \\
2.33 \\
2.34 \\
2.18\end{array}$ & $\begin{array}{l}2.458 \\
2.368 \\
2.465 \\
2.33 \\
2.587 \\
2.44\end{array}$ & $\begin{array}{l}1.02 \\
1.00 \\
1.14 \\
\\
1.08 \\
1.07\end{array}$ & $\begin{array}{l}\mathrm{G}_{\mathrm{a}}(\mathrm{r}) \text { this work } \\
\mathrm{G}_{\text {eos }}(\mathrm{r}) \text { this work } \\
\text { expt., ref. } 12 \mathrm{a} \\
\text { ref. } 4 \mathrm{~b} \\
\text { ref. } 13 \\
\text { ref } 14\end{array}$ \\
\hline $\mathrm{C}_{12}$ & $\begin{array}{l}1.79 \\
1.76 \\
1.76 \\
1.84\end{array}$ & $\begin{array}{l}1.624 \\
1.524 \\
1.473 \\
1.54 \\
1.412 \\
1.49\end{array}$ & $\begin{array}{l}0.694 \\
0.683 \\
0.619 \\
\\
0.68 \\
0.652\end{array}$ & $\begin{array}{l}\mathrm{G}_{\mathrm{a}}(\mathrm{r}) \text { this work } \\
\mathrm{G}_{\text {eos }}(\mathrm{r}) \text { this work } \\
\text { expt., ref. } 12 \mathrm{a} \\
\text { ref. } 4 \mathrm{~b} \\
\text { ref. } 13 \\
\text { ref } 14\end{array}$ \\
\hline $\mathrm{C}_{44}$ & $\begin{array}{l}0.707 \\
0.705 \\
0.712 \\
0.65\end{array}$ & $\begin{array}{l}1.215 \\
1.214 \\
1.247 \\
1.28 \\
1.299 \\
1.26\end{array}$ & $\begin{array}{l}0.351 \\
0.349 \\
0.316 \\
\\
0.45 \\
0.322\end{array}$ & $\begin{array}{l}\mathrm{G}_{\mathrm{a}}(\mathrm{r}) \text { this work } \\
\mathrm{G}_{\mathrm{eos}}(\mathrm{r}) \text { this work } \\
\text { expt., ref. 12a } \\
\text { ref. } 4 \mathrm{~b} \\
\text { ref. } 13 \\
\text { ref. } 14\end{array}$ \\
\hline $\mathrm{E}_{\mathrm{uvf}}$ & $\begin{array}{l}1.518 \\
1.527 \\
1.4 \\
1.44\end{array}$ & $\begin{array}{l}1.70 \\
1.69 \\
1.51 \\
1.63 \\
1.51 \\
1.60 \\
1.62\end{array}$ & $\begin{array}{l}0.74 \\
0.74 \\
0.75 \\
\\
\\
0.64 \\
0.73 \\
0.7\end{array}$ & $\begin{array}{l}\mathrm{G}_{\mathrm{a}}(\mathrm{r}) \text { this work } \\
\mathrm{G}_{\text {eos }}(\mathrm{r}) \text { this work } \\
\text { expt., ref. } 12 \mathrm{~b}-\mathrm{d} \\
\text { ref. } 4 \mathrm{~b} \\
\text { ref. } 13 \\
\text { ref. } 14 \\
\text { ref } 15\end{array}$ \\
\hline $\mathrm{E}_{\mathrm{usf}}$ & $\begin{array}{l}192 \\
192 \\
180\end{array}$ & $\begin{array}{l}116 \\
116 \\
125 \\
115\end{array}$ & $\begin{array}{l}139 \\
139 \\
135\end{array}$ & $\begin{array}{l}\mathrm{G}_{\mathrm{a}}(\mathrm{r}) \text { this work } \\
\mathrm{G}_{\text {eos }}(\mathrm{r}) \text { this work } \\
\text { expt., ref. } 20 \\
\text { ref. } 16\end{array}$ \\
\hline
\end{tabular}


Table IV.

Convergence test for computational cell size using palladium functions.

\begin{tabular}{|c|c|c|c|c|c|c|}
\hline $\begin{array}{l}\text { type of } \\
\text { defect }\end{array}$ & $\mathfrak{j}_{\mathbf{K}}^{\text {cell size }}$ & & $\begin{array}{l}\text { number } \\
\text { of atoms }\end{array}$ & $\begin{array}{l}\text { steps }^{\mathrm{a}} \\
\text { required }\end{array}$ & $\begin{array}{c}\mathrm{cpu}^{\mathrm{b}} \\
(\mathrm{msec} / \mathrm{step})\end{array}$ & $\begin{array}{l}\text { Defect } \\
\text { Energy }\end{array}$ \\
\hline $\begin{array}{l}\text { single } \\
\text { vacancy }\end{array}$ & $\begin{array}{ll}3 & 3 \\
4 & 4 \\
5 & 5 \\
6 & 6\end{array}$ & $\begin{array}{l}3 \\
4 \\
5 \\
6\end{array}$ & $\begin{array}{l}107 \\
255 \\
499 \\
863\end{array}$ & $\begin{array}{r}85 \\
124 \\
168 \\
206\end{array}$ & $\begin{array}{r}18 \\
41 \\
81 \\
140\end{array}$ & $\begin{array}{l}1.40 \\
1.39 \\
1.39 \\
1.39\end{array}$ \\
\hline
\end{tabular}

self-

interstitial

$\begin{array}{llllr}3 & 3 & 3 & 109 & 94 \\ 4 & 4 & 4 & 257 & 142\end{array}$

$94 \quad 18$

$142 \quad 42$

3.31

$\begin{array}{lllll}5 & 5 & 5 & 501 & 207\end{array}$

$\begin{array}{lllll}6 & 6 & 6 & 865 & 267\end{array}$

81

3.00

$(100)$

$\begin{array}{llll}4 & 4 & 4 & 256\end{array}$

666

864

275

$\begin{array}{lll}4 & 4 & 8\end{array}$

512

373

189

$4 \quad 410$

640

467

141

2.99

(110)

$\begin{array}{llll}4 & 4 & 4 & 128\end{array}$

$\begin{array}{llll}5 & 5 & 5 & 250\end{array}$

194

264

345

$8 \begin{array}{lll}8 & 8 & 8\end{array}$

432
1024

484

(111)

surface

$\begin{array}{llll}4 & 4 & 4 & 384 \\ 4 & 4 & 6 & 576\end{array}$

114

473

961

$\begin{array}{llll}4 & 4 & 8 & 786\end{array}$

$4 \quad 412$

1152

2117

3681

intrinsic-

stacking-

$\begin{array}{llll}4 & 4 & 4 & 384\end{array}$

$\begin{array}{lll}4 & 4 & 8\end{array}$

786

790

2607

4881

35
124

1567

76

1567

1567

$100 \quad 1567$

fault

$4 \quad 412 \quad 1152$

1917

3736

5352

141

1674

$34 \quad 1674$

$60 \quad 1674$

$146 \quad 1674$

coherent-

twin-

boundary

$\begin{array}{rrrrr}4 & 4 & 8 & 786 \\ 4 & 4 & 2 & 1152 \\ 4 & 4 & 16 & 1536\end{array}$

$\begin{array}{rr}55 & 1476 \\ 86 & 1476 \\ 116 & 1476 \\ 180 & 1476 \\ 238 & 1476\end{array}$

55

116

180

172

172

172

$116 \quad 83.3$

$180 \quad 89.1$

$238 \quad 89.1$

\footnotetext{
a Steps required for VQEMM with an energy convergence criterion of $1.0 \times 10^{-6} \mathrm{eV}$.

b The cpu time in milliseconds per step using the SRL/CRAY X-MP/132.

c Units are $\mathrm{eV}$ for point defects and $\mathrm{erg} / \mathrm{cm}^{2}$ for planar defects.
} 


\section{Table $\mathbf{V}$.}

Calculated Energies for Defects.

Where two numbers are given the top number is calculated with these functions and the lower is the experimental value.

$\begin{array}{lcllc} & \text { Pd } & \mathrm{Ni} & \mathrm{Al} & \text { reference } \\ \mathrm{E}_{\text {vf }} & 1.4 & 1.7 & 0.72 & \\ & 1.4 & 1.6 & 0.75 & 12 \\ \mathrm{E}_{\text {sif }} & 3.0 & 4.1 & 1.7 & \\ \mathrm{E}_{(100)} & 1567 & 1780 & 722 & \\ & & 1821 \pm 182 & & 19 \\ \mathrm{E}_{(110)} & 1674 & 1944 & 760 & \\ & & 1900 \pm 190 & & 19 \\ \mathrm{E}_{(111)} & 1476 & 1671 & 697 & \\ \text { Experimental } & 2000 & 1820 \pm 180 & 1140 & 19 \\ \text { (Surface Tension) } & & & & \\ \mathrm{E}_{\text {sf }} & 173 & 108 & 119 & \\ & 180 \pm 30 & 125 \pm 25 & 135 \pm 30 & 20 \\ \mathrm{E}_{\text {tb }} & 89 & 55 & 63 & \end{array}$




\section{Table VI.}

Structural relaxation at surfaces and planar defects.

Surface relaxation of the top-layer spacing $\mathrm{Dz}_{12}$ and the second layer spacing $\mathrm{Dz}_{23}$, for the lowindex fr ces. Relaxation at planar defects measures changes in layer-spacing for the first inter-planar spacing, near the defect $\mathrm{Dz}_{12}$ and the second layer spacing $\mathrm{Dz}_{23}$. Distances are in $\AA$.

$\mathbf{P d}$

$\mathrm{Ni}$

Al

(100) surface

$\begin{array}{lllr}\mathrm{Dz}_{12} & -0.043 & -0.0065 & -0.041 \\ \mathrm{Dz}_{23} & +0.004 & +0.0012 & +0.004\end{array}$

(110) surface

$\begin{array}{lllr}\mathrm{Dz}_{12} & -0.12 & -0.042 & -0.13 \\ \mathrm{Dz}_{23} & +0.03 & +0.008 & +0.05\end{array}$

(111) surface

$\begin{array}{llll}\mathrm{Dz}_{12} & -0.051 & -0.0054 & -0.015 \\ \mathrm{Dz}_{23} & -0.001 & -0.0022 & +0.003\end{array}$

intrinsic stacking fault

$\begin{array}{llll}\mathrm{Dz}_{12} & +0.050 & +0.025 & +0.083 \\ \mathrm{Dz}_{23} & +0.020 & +0.012 & +0.032\end{array}$

coherent twin boundary

$\begin{array}{lllrl} & \mathrm{Dz}_{12} & +0.023 & +0.012 & +0.036 \\ \mathrm{Dz}_{23} & -0.003 & -0.001 & -0.006 & \end{array}$


